\title{
Analgesia in patients undergoing thoracotomy: Epidural versus paravertebral technique. A randomized, double-blind, prospective study
}

\author{
Federico Raveglia, MD, ${ }^{a}$ Alessandro Rizzi, MD, ${ }^{a}$ Andrea Leporati, MD, ${ }^{a}$ Piero Di Mauro, MD, ${ }^{b}$ \\ Ugo Cioffi, $\mathrm{PhD},{ }^{\mathrm{c}}$ and Alessandro Baisi, $\mathrm{MD}^{\mathrm{a}}$
}

\begin{abstract}
Background: Pain control after thoracotomy prevents postsurgical complications and improves respiratory function. The gold standard for post-thoracotomy analgesia is the epidural catheter. The aim of this study was to compare it with a new technique that involves placement of a catheter in the paravertebral space at the end of surgery under a surgeon's direct vision.
\end{abstract}

\begin{abstract}
Methods: From November 2011 to June 2012, 52 patients were randomized into 2 groups depending on catheter placement: an epidural catheter for group A and a paravertebral catheter for group B. At 12, 24, 48, and 72 hours after surgery, the following parameters were recorded: (1) pain control using the patient's completion of a visual analog scale module, (2) respiratory function using forced expiratory volume in 1 second and ambient air saturation, and (3) blood cortisol values as an index of systemic reaction to pain.
\end{abstract}

\begin{abstract}
Results: Statistically significant differences $(P<.05)$ were found in favor of group B for both cough and rest pain control $(P=.002$ and .002 , respectively) and respiratory function in terms of forced expiratory volume in 1 second and ambient air saturation levels $(P=.023$ and .001 , respectively). No statistically significant differences were found in blood cortisol trends between the 2 groups $(P>.05)$. Collateral effects such as vomiting, nausea, low pressure, or urinary retention were observed only in group A. No collateral effects were recorded in the paravertebral group.
\end{abstract}

Conclusions: According to our data, drugs administered through a paravertebral catheter are very effective. Moreover, it does not present contraindications to its positioning or collateral effects. More studies are necessary to confirm data we collected. (J Thorac Cardiovasc Surg 2014;147:469-74)

Pain after standard thoracotomy is often present and associated with severe complications, such as atelectasis. This can also develop into a severe pneumonia due to retention of secretions. $^{1-3}$ Pain prevents effective coughing, deep breathing, and a patient's mobility. Generally, strong pain after surgery increases perioperative morbidity and may also lead to chronic pain. ${ }^{4-6}$ At present, various techniques are proposed and used to prevent thoracic pain after thoracotomy. Among these, the most common is thoracic epidural anesthesia (EA), considered to be the gold standard. ${ }^{7-9}$

Our study compares the efficacy of EA with a technique that consists of the placement of a catheter in the

\footnotetext{
From the Department of Thoracic Surgery, ${ }^{a}$ A.O. San Paolo, Intensive Care Unit, ${ }^{\text {b }}$ A.O. San Paolo, and IRCCS Ospedale Maggiore, ${ }^{c}$ University of Milan, Milan, Italy.

Disclosures: Authors have nothing to disclose with regard to commercial support.

Read at the 93rd Annual Meeting of The American Association for Thoracic Surgery, Minneapolis, Minnesota, May 4-8, 2013.

Received for publication May 7, 2013; revisions received Aug 7, 2013; accepted for publication Sept 4, 2013; available ahead of print Nov 4, 2013.

Address for reprints: Federico Raveglia, MD, University of Milan, Antonio di Rudinì St, Number 8, 20142 Milan, Italy (E-mail: federico.raveglia@ao-sanpaolo.it). $0022-5223 / \$ 36.00$

Copyright (C) 2014 by The American Association for Thoracic Surgery http://dx.doi.org/10.1016/j.jtcvs.2013.09.024
}

paravertebral space, resulting in paravertebral anesthesia (PA).

This technique was previously proposed and tested almost 20 years ago but never became very popular in clinical practice. ${ }^{10,11}$ The most interesting and recent article on this topic presents a systemic review and meta-analysis of 10 randomized trials by Davies and colleagues, ${ }^{12}$ including 520 adult patients. PA resulted the same in terms of pain control, but was better as concerns contraindications and adverse effects in comparison with EA. However, the studies were of moderate quality because they did not use uniform populations regarding positioning techniques, drugs used, and largely because there were no blinding.

An EA catheter is usually placed by an anesthesiologist immediately before surgery when the patient is awake, using local anesthesia to prevent positioning pain. This method is contraindicated for patients taking anticoagulant or antiplatelet drugs that cannot be suspended for the perioperative period or for those who have coagulopathies. ${ }^{13,14}$ Moreover, this technique may result in dangerous risks during placement, including dural perforation, spinal cord hematoma, spinal infection, or abscess. ${ }^{15,16}$ During treatment some adverse effects may occur, including hypotension, urinary retention, nausea, vomiting, or itching. ${ }^{17-19}$ On the contrary, the PA catheter does not 


\section{Abbreviations and Acronyms}

EA $=$ epidural anesthesia

FEV1 = forced expiratory volume in 1 second

$\mathrm{PA}=$ paravertebral anesthesia

VAS $=$ visual analog scale

present any contraindications during placement and, due to the drugs used and to the anatomical space where they are administered, it has no side effects. Two different approaches for placing the catheter in the paravertebral space are used: a blind approach, also known as an anesthetic approach, using the loss of resistance technique first described by Eason and Wyatt, ${ }^{17}$ and a de visu approach where the catheter is placed by a surgeon at the end of the thoracotomy. ${ }^{18}$

The aim of our study was to investigate if PA is as effective as EA in patients undergoing thoracotomy. Our primary outcome was to compare pain control, both at rest and while coughing, between the 2 groups. Our secondary outcome was to compare surgical stress and respiratory function in these patients.

\section{MATERIALS AND METHODS}

The study was approved by the local ethics committee of St Paolo Hospital at the University of Milan (No. 9898). All recruited patients provided informed and written consent to the study. We considered patients who underwent muscle-sparing thoracotomy for surgery due to pulmonary neoplastic diseases, pleuric empyema, lung volume reduction surgery, bronco-pleural fistula, or infectious diseases. Our inclusion and exclusion criteria are reported in Table 1. The study was prospective, randomized, and double-blind.

Patients were recruited between November 2011 and June 2012 and randomly located by computer-generated randomization in 1 of the following 2 groups: Group A, EA with infusion through the catheter of $0.001 \%$ fentanyl $(10 \mu \mathrm{g} / \mathrm{mL})$ with $0.1 \%$ bupivacaine. Group B, PA with infusion of $0.3 \%$ naropine $(5-10 \mathrm{~mL}$ vials $10 \mathrm{mg} / \mathrm{mL}$ in $100 \mathrm{cc}$ $0.9 \%$ saline solution). Each patient in the 2 groups had simultaneous infusion of paracetamol (1-500 mg vial) 4 times a day and the opportunity to ask for tramadol $(1-50 \mathrm{mg} / 1 \mathrm{~mL}$ vial in $100 \mathrm{cc}$ of $0.9 \%$ saline if visual analog scale (VAS) score was $>6$ maximum twice a day). Any other requests by patients to be administered more pain medication were recorded and satisfied.

All patients were pretreated with sublingual morphine. In group A the epidural catheter was placed immediately before surgery according to the standard techniques. The patient was awake and placed in a seated position and the interspace T5/6, T6/7 was detected; using the midline approach and the loss of resistance technique, the catheter was inserted. In group B the paravertebral catheter was placed at the end of surgery using the de visu technique: an 18-gauge Thohy needle was placed through the chest wall at an appropriate site in the same interspace as the thoracotomy incision. The needle's obturator was removed and the catheter passed through and emerged inside the thoracic cavity. A localized extrapleural, paravertebral pocket was then created by placing a gently curved clamp under the parietal pleura at the posterior end or apex of the intercostal incision. Then, the catheter was gently prompted inside the pleural pocket and pushed close to the paravertebral space. Once positioned, a piece of hemostatic sponge was placed at the entrance of the pleural pocket to avoid spreading of medication. The external side of the catheter was then fixed to the skin with a transparent patch. We always placed 2 chest tubes before thoracotomy closure. All surgeries were performed by AB and FR. In each group the catheter was removed on the same day that the final chest tube was removed, between the third and fifth day after surgery, except for 1 patient with prolonged air leaks.

We arbitrarily identified the length of surgery as the period from the arrival of patients in the operating room area to the end of thoracotomy closure. To evaluate pain systemic response blood cortisol was measured 30 minutes after thoracotomy and then at 6, 12, 24, 48, and 72 hours after surgery. Pain level was measured using a VAS, where $0=$ indicates no pain and $10=$ severe pain, at $6,12,24,48$, and 72 hours after surgery. To evaluate pulmonary function at $6,12,24,48$, and 72 hours, forced expiratory volume in 1 second (FEV1) and ambient air saturation were measured. Possible drug-related complications such as urinary retention (defined as Foley catheter replacement after initial removal), itching, nausea/vomiting, or postural hypotension were recorded in the postoperative period. We evaluated pain using the VAS scale, both at rest and while coughing, during the 3 months postsurgical clinical control, and recorded the patients' VAS scale answers. All data were recorded by the research fellow (ie, AR). Normally distributed results were compared by Student $t$ test analysis and nonnormally distributed results were compared by Mann-Whitney analysis. The population of the recruited patients was calculated to be sufficient for obtaining statistical significance.

\section{RESULTS}

From November 2011 to June 2012, 52 patients were enrolled and randomized for the study. Three patients were excluded from the EA group due to an erroneous location of the catheter and 1 patient was excluded from the PA group because of accidental removal of the catheter. The 2 groups were composed of 24 patients each, $50 \%$ and $54 \%$ men in the EA and PA groups, respectively. The mean patient age was 78 years in both groups. No statistical significance was found in the demographic traits (ie, weight, height, and body mass index) of the 2 groups $(P>.468)$ (Table 2). No patients in either group had a prior history

TABLE 1. Inclusion and exclusion criteria

\begin{tabular}{ll}
\hline \multicolumn{1}{c}{ Inclusion criteria } & \multicolumn{1}{c}{ Exclusion criteria } \\
\hline Age $>18$ or $<80 \mathrm{y}$ & Age $<18$ or $>80 \mathrm{y}$ \\
Karnofsky performance scale $\geq 70 \%$ & Coagulopathies \\
American Society of Anesthesiology & Therapies \\
$\quad$ Classification $<\mathrm{IV}$ & \\
Forced expiratory volume in 1 second & Allergies \\
$\geq 50 \%$ predicted & \\
Wegener's granulomatosis (white blood cell & Spinal deformities \\
$\quad$ count $>4000 / \mathrm{mm}^{3}$ ) & \\
Primary systemic chemotherapy (platelet & Neurologic diseases \\
$\quad$ count $>100,000 / \mathrm{mm}^{3}$ ) & Psychiatric diseases \\
Hemoglobin $>8.5 \mathrm{~g} / \mathrm{dL}$ & Past thoracic surgery \\
Bilirubin $<3.0 \mathrm{mg} / \mathrm{dL}$ & Pre-op thoracic drainage \\
Aspartate transaminase $<2$ times limits & Past acute myocardial \\
Creatinine $<3.0 \mathrm{mg} / \mathrm{dL}$ & infarction \\
Carbon dioxide tension $<50 \mathrm{~mm} \mathrm{Hg}$ & Abuse of alcohol or drugs \\
& Body mass index $>30$ \\
& Pregnancy \\
\hline Pre-op, Preoperative
\end{tabular}

Pre-op, Preoperative. 
TABLE 2. Demographic traits of participants

\begin{tabular}{lccc}
\hline Demographic characteristic & Total $(\mathbf{N}=\mathbf{4 8})$ & Group A $(\mathbf{n}=\mathbf{2 4})$ & Group B (n= 24) \\
\hline Sex, men & $25(52.08)$ & $12(50.0)$ & $13(54.2)$ \\
Age, y & $78.54 \pm 7.787(80.00[51-92])$ & $78.63 \pm 5.523(80.00[65-89])$ & $78.46 \pm 9.664(80.5[51-92])$ \\
Weight, $\mathrm{kg}$ & $78.02 \pm 10.20(72.00[52-100])$ & $76.92 \pm 11.36(74.50[52-100])$ & $79.13 \pm 8.994(79.50[62-98])$ \\
Height, cm & $168.96 \pm 5.149(169.5[158-180])$ & $168.75 \pm 5.007(170.0[159-180])$ & $169.17 \pm 5.387(169.0[158-180])$ \\
Body mass index & $27.3518 \pm 3.517(27.20[18.21-33.9])$ & $27.050 \pm 4.067(27.30[18.21-33.91])$ & $27.653 \pm 2.924(27.20[21.71-32.79])$ \\
\hline
\end{tabular}

Values are presented as $\mathrm{n}(\%)$ or mean \pm standard deviation (median [min-max]).

of thoracic surgery and none had known allergies. All patients had surgeries for lung cancer, except for 1 who had surgery for a lung abscess.

The length of surgery was 141.3 minutes for the EA group and 108.6 minutes for the PA group with a statistical significance in favor of the PA group $(P<.0001)$ (Table 3$)$. There were no recorded adverse effects in the PA group, in contrast with the EA group, in which we found 9 out of 24 $(37.5 \%)$ patients with postural hypotension, 6 out of 24 $(25 \%)$ patients with urinary retention, 6 out of $24(25 \%)$ with itching, and 8 out of $24(33.3 \%)$ with nausea or vomiting (Table 3). No mortality was reported. The only surgical complication was 1 patient with prolonged air leaks. In the 2 groups there was no difference in terms of hospital length of stay.

FEV1 measurements were taken preoperatively and at 6 , $12,24,48$, and 72 hours after the operation with statistical significance $(P=.023)$ in favor of the PA group. VAS was recorded at $6,12,24,48$, and 72 hours after operation both at rest and while coughing with statistical significance in favor of the PA group $(P=.002)$. Ambient air saturation was taken at $6,12,24,48$, and 72 hours after the operation with statistical significance in favor of PA group $(P=.023)$. Cortisol blood levels were measured at 30 minutes after the surgery and then at $6,12,24,48$, and 72 hours after operation with no statistical significance $(P=.08)$ among the 2 groups (Table 4$)$. Data were then also exactly analyzed, comparing variables at each moment of recording. VAS records, at rest and while coughing, always presented statistical significance in favor of the PA group at 6, 12, 24, 48, and 72 hours after the surgery. Ambient air saturation and FEV1 presented a statistical significance in favor of the PA group at 24, 48, and 72 hours after the surgery. Cortisol level records did not have a statistically significant variance at any time.

None of the patients in either groups required additional pain medication.
Three months after surgery there was no statistical difference in pain between the 2 groups.

\section{DISCUSSION}

Pain is the most important factor responsible for disorders in a patient's respiratory mechanism and pulmonary function in the perioperative period after thoracic surgery. ${ }^{1-6}$ It has different origins, making its etiology very complex. Pain impulses originating from stimuli from the chest wall and parietal pleura pass along the intercostal nerves; in addition, those originating from diaphragmatic pleura ascend within the afferent fibers within the phrenic nerves, and those from the lung and mediastinum are carried by the vagus nerve. Surgical techniques influence the nature and intensity of postoperative pain. Standard thoracotomy is more painful than limited thoracotomy and videoassisted thoracoscopic surgery. ${ }^{19}$ We adopted a musclesparing incision that is a fairly conservative thoracotomy. ${ }^{20}$

In particular, in the case of thoracotomy, retraction on the posterior spinal muscles determines stimuli transmitted by the posterior primary branches of the spine and the incision pain is mediated by the anterior branches. Moreover, sympathetic nerves mediate pain from the visceral pleura, lung, and neuro-humoral factors. All of these pathways establish stimuli for the origin of pain and must be blocked to dominate pain.

Following thoracic surgery, a restrictive respiratory pathway develops, which decreases to approximately $40 \%$ the baseline values of FEV1, forced vital capacity, and functional residual capacity. The relationship between functional residual lung capacity and closing capacity predicts if atelectasis will develop; a decreasing functional residual lung capacity to a value less than closing capacity leads to airway narrowing or closure, which produces an area with a low ventilation perfusion relationship. Simultaneously, postoperative pain highly limits voluntary deep

TABLE 3. Perioperative characteristics of participants

\begin{tabular}{|c|c|c|c|c|}
\hline Characteristic & Total $(N=48)$ & Group A $(n=24)$ & Group B $(n=24)$ & $P$ \\
\hline Length of surgery, min & $124.9 \pm 19.61(122.5[95-154])$ & $141.3 \pm 10.09(146.0[118-154])$ & $108.6 \pm 11.170(108.0[95-140])$ & $<.0001^{*}$ \\
\hline Allergy (no) & $48(100.0)$ & $24(100.0)$ & $24(100.0)$ & 1.00 \\
\hline Hypotension (yes) & $9(18.75)$ & $9(37.5)$ & $0(0)$ & $0.002^{*}$ \\
\hline Urinary retention (yes) & $6(12.50)$ & $6(25)$ & $0(0)$ & $0.022 *$ \\
\hline Vomiting/nausea (yes) & 8 (16.66) & $8(33.3)$ & $0(0)$ & $0.004^{*}$ \\
\hline Hitch (yes) & $6(12.50)$ & $6(25)$ & $0(0)$ & $0.022 *$ \\
\hline
\end{tabular}

Data are presented as mean \pm stardard deviation (median $[\min -\max ])$ or $\mathrm{n}(\%)$. $* P<.05$. 
TABLE 4. Overall results

\begin{tabular}{|c|c|c|c|c|c|c|c|}
\hline Parameter & $-24 h$ & $6 \mathrm{~h}$ & $12 \mathrm{~h}$ & $24 \mathrm{~h}$ & $48 \mathrm{~h}$ & $72 \mathrm{~h}$ & $\boldsymbol{P}$ \\
\hline \multirow[t]{2}{*}{ Visual analog scale } & - & $5.92 \pm 1.269$ & $4.62 \pm 1.540$ & $3.60 \pm 1.572$ & $2.98 \pm 1.545$ & $2.78 \pm 1.608$ & $.002 *$ \\
\hline & - & $6.00(2-8)$ & $5.00(1-7)$ & $4.00(1-7)$ & $3.00(1-7)$ & $2.00(1-7)$ & \\
\hline \multirow[t]{2}{*}{ Vas cough } & - & $6.10 \pm 1.276$ & $4.79 \pm 1.641$ & $3.62 \pm 1.556$ & $3.09 \pm 1.607$ & $2.87 \pm 1.687$ & $.002 *$ \\
\hline & - & $6.00(2-9)$ & $5.00(1-8)$ & $4.00(1-7)$ & $3.00(1-7)$ & $2.00(1-7)$ & \\
\hline \multirow[t]{2}{*}{ Cortisol } & - & $16.33 \pm 4.400$ & $13.74 \pm 3.564$ & $11.85 \pm 3.789$ & $9.42 \pm 2.387$ & $8.00 \pm 2.456$ & .08 \\
\hline & - & $18.00(5-22)$ & $14.00(6-22)$ & $11.00(5-20)$ & $9.90(5-16)$ & $8.00(4-14)$ & \\
\hline \multirow[t]{2}{*}{ Saturation } & $96.77 \pm 1.387$ & $91.35 \pm 3.049$ & $94.26 \pm 2.059$ & $94.93 \pm 1.959$ & $96.13 \pm 1.546$ & $96.64 \pm 1.264$ & $.001 *$ \\
\hline & $97.00(93-99)$ & $92.00(81-96)$ & $95.00(89-98)$ & $95.00(90-98)$ & $96.00(93-99)$ & $96.00(95-99)$ & \\
\hline \multirow{2}{*}{$\begin{array}{l}\text { Forced expiratory volume } \\
\text { in } 1 \text { second }\end{array}$} & $79.63 \pm 10.724$ & - & $47.62 \pm 10.233$ & $52.53 \pm 11.918$ & $56.27 \pm 10.672$ & $60.67 \pm 9.888$ & $.023^{*}$ \\
\hline & $83.00(51-95)$ & - & $45.00(33-76)$ & $50.00(39-79)$ & $57.00(39-81)$ & $61.00(40-82)$ & \\
\hline
\end{tabular}

Data are presented as mean \pm standard deviation or median (min-max). $* P<.05$.

breathing, coughing, and patient mobility, all necessary to break down atelectasis. All of these conditions can be associated with respiratory infection. ${ }^{20}$ Many modalities for the management of post-thoracotomy pain are known. Intravenous narcotic drugs may work adequately, but are related to cough suppression, as well as central nervous system and respiratory depression, leading once again to retention of secretions and atelectasis. To limit the effects of systemic narcotics, EA has been used extensively and is considered to be the gold standard for pain relief after thoracic surgery. ${ }^{7-9}$ This technique allows direct anesthetization of the spinal branches and sympathetic nerves using less narcotic drugs with excellent control of pain and decreased respiratory and central nervous system depression. ${ }^{5,6}$

EA presents several intrinsic contraindications that are absolute (eg, coagulopathy, local sepsis, allergy to amide local anesthetics, and anatomical anomalies) and relative (activated partial thromboplastin time ratio or international normalized ratio 1.2-1.4). ${ }^{21}$ Technical difficulties with insertion have been reported in up to $11 \%$ of patients. Bilateral effects of local anesthetic on the sympathetic chain have been associated with increased rates of hypotension, itching, vomiting/nausea, urinary retention, and sometimes mental status changes and respiratory depression have been observed.

PA technique presents several advantages in comparison with EA. It does not have contraindications concerning coagulopathies or anatomical anomalies and is free of any systemic collateral effects because only local anesthetics are used. The PA is placed at the end of surgery, whereas patients are still under anesthesia, through a safe and easy to learn surgical technique.

Our results show that there has always been a significant difference in favor of PA as far as pain management is concerned, both at rest and while coughing, in the 72 hours after surgery. Data also proves that PA is more effective than EA concerning respiratory outcomes both for pulmonary function in terms of FEV1 $(P=.023)$ and ambient air saturation $(P=.023)$.
Concerning its placement, a PA catheter presents convenient advantages. It is an intraoperative maneuver performed by surgeons in 4 to 5 minutes, reducing the patient's stay in the operating room area. It should also be emphasized that PA is suitable for patients in whom an EA catheter was not placed because the procedure started in video-assisted thoracoscopic surgery and then converted to a thoracotomy. It is remarkable that our results demonstrate the absence of adverse effects in the PA group suggesting minor affects on this technique.

The strength of our study lies in the prospective randomization nature and the collection of objective data, such as FEV1, blood cortisol level, and saturation and oxygen tension level. The most important limitations are represented by the limited number of patients recruited, even though sufficient to reach statistical significance, and the influence of patients' personal evaluation of pain.

\section{CONCLUSIONS}

The PA catheter is safe and effective and should be always considered as an EA catheter alternative. In any case, the PA and catheter placement technique should be known by all thoracic surgeons.

This research won the Romeo and Enrica Invernizzi award. The authors thank Dr Radaelli Giovanni for providing statistical assistance in the preparation of this manuscript.

\section{References}

1. Ochroch EA, Gottschalk A, Augostides J, Carson KA, Kent L, Malayaman N, et al. Long-term pain and activity during recovery from major thoracotomy using thoracic epidural analgesia. Anesthesiology. 2002;97:1234-44.

2. Senturk M, Ozcan PE, Talu GK, Klyan E, Camci E, Ozyalcin S, et al. The effects of three different analgesia techniques on long-term post thoracotomy pain. Anesth Analg. 2002;94:11-5.

3. Muehling BM, Halter GL, Schelzig H, Meierhenrich R, Steffen P, SunderPlassmann L, et al. Reduction of post-operative pulmonary complications after lung surgery using a fast track clinical pathway. Eur J Cardiothoracic Surg. 2008;34:174-80.

4. Dowling R, Thielmeier K, Ghaly A, Barber D, Boice T, Dine A. Improved pain control after cardiac surgery: results of a randomized, double-blind, clinical trial. J Thorac Cardiovasc Surg. 2003;126:1271-8. 
5. Wildgaard K, Ravn J, Kehlet H. Chronic post-thoracotomy pain: a critical review of pathogenic mechanism and strategies for prevention. Eur J Cardiothorac Surg. 2009;36:170-80.

6. Kehlet H, Dahl JB. Anesthesia, surgery, and challenges in postoperative recovery. Lancet. 2003;362:1921-8.

7. Bakhtiary F, Therapidis P, Dzemail O, Ak K, Ackermann H, Meninger D, et al. Impact of high thoracic epidural anesthesia on incidence of perioperative atrial fibrillation in off-pump coronary bypass grafting: a prospective randomized study. J Thorac Cardiovasc Surg. 2007;134:460-4

8. Lubenow TR, Faber LP, McCarthy RJ, Hopkins EM, Warren WH, Ivankovich $\mathrm{AD}$. Post-thoracotomy pain management using continuous epidural analgesia in 1324 patients. Ann Thorac Surg. 1994;58:924-30.

9. Kaiser AM, Zollinger A, De Lorenzi D, Largiarder F, Weder W. Prospective, randomized comparison of extra pleural versus epidural analgesia for postthoracotomy pain. Ann Thorac Surg. 1998;66:367-72

10. Deneuville M, Bisserier A, Regnard JF, Chevalier M, Levasseur P, Herve P. Continuous intercostal analgesia with $0.5 \%$ bupivacaine after thoracotomy: a randomized study. Ann Thorac Surg. 1993;55:381-5

11. Joshi GP, Bonnet F, Shah R, Wilkinson RC, Camu F, Fischer B, et al. A systematic review of randomized trials evaluating regional techniques for postthoracotomy analgesia. Anesth Analg. 2008;107:1026-40.

12. Davies RG, Myles PS, Graham JM. A comparison of the analgesic efficacy and side-effects of paravertebral vs epidural blockade for thoracotomy — a systematic review and meta-analysis of randomized trials. Br J Anaesth. 2006;96:418-26.

13. Dajczman E, Gordon A, Kreisman H, Wolkove N. Long-term post-thoracotomy pain. Chest. 1991;99:270-4

14. Tiipana E, Nilsson E, Kalso E. Post-thoracotomy pain after thoracic epidural analgesia: a prospective follow-up study. Acta Anaesthesiol Scand. 2003;47:433-8.

15. Soto RG, Fu ES. Acute pain management for patients undergoing thoracotomy. Ann Thorac Surg. 2003;75:1349-57.

16. Matthews PJ, Govenden V. Comparison of continuous paravertebral and extradural infusion of bupivacaine for pain relief after thoracotomy. Br J Anaesth. 1989;62:204-5.

17. Eason MJ, Wyatt R. Paravertebral thoracic block - a reappraisal. Anaesthesia. 1979;34:638-42.

18. Bimston DN, McGee JP, Liptay MJ, Fry WA. Continuous paravertebral extra pleural infusion for post-thoracotomy pain management. Surgery. 1999;126: 650-6; discussion 656-7.

19. Landreneau RJ, Pigula F, Luketich JD, Keenan RJ, Bartley S, Fetterman LS, et al. Acute and chronic morbidity differences between muscle-sparing and standard lateral thoracotomies. J Thorac Cardiovasc Surg. 1996;112:1346-50; discussion 1350-1.

20. Nosotti M, Baisi A, Mendogni P, Palleschi A, Tosi D, Rosso L. Muscle sparing versus posterolateral thoracotomy for pulmonary lobectomy: randomized controlled trial. Interact Cardiovasc Thorac Surg. 2010;11:415-9.

21. Hyllested M, Jones S, Pedersen JL, Kehlet H. Comparative effect of Paracetamol, NSAIDs or their combination in post-operative pain management: a qualitative review. Br J Anaesth. 2002;90:166-72.

\section{Discussion}

Dr Arjun Pennathur (Pittsburgh, Pa). At the University of Pittsburgh we published a randomized study of epidural versus paravertebral catheters about 10 years ago. What we found was equal pain control between the 2 groups, but the Foley catheter patients who had an epidural catheter needed it longer.

So my question is regarding the design of the study. Did you standardize who placed the epidural catheter? Epidural catheter placement is a technical thing and there are differences between anesthesiologists who place it and its effectiveness. Do you know how to standardize your epidural catheter arm? Is it done by a group of anesthesiologists specializing in pain control, for example?

And the second thing is for many of the pain-associated symptoms, it is also believed that the epidural catheter should be placed before the incision similar to the paravertebral catheter being placed before the actual incision and the trauma of the surgery when you measure the mediators and so forth.

I was wondering if you showed an intraoperative picture of placing a paravertebral catheter, but in your randomization scheme, did you consider placing the paravertebral catheter preoperatively, before actually making the skin incision?

Dr Federico Raveglia (Milan, Italy). The anesthesiologist who performed the procedure was always the same, and the anesthesiologists involved were all skilled in doing that procedure. So I think that the position of the epidural catheter was always the same.

Concerning the paravertebral catheter, it is always the same to do that, so I think the 2 groups were pretty standardized.

Dr Harold M. Burkhart (Rochester, Minn). He also asked if you considered putting the paravertebral catheter in before the incision.

Dr Raveglia. The paravertebral catheter is placed before the incision because it used to be placed with the patients awake to start the treatment before the issue itself. It's more effective.

The choice to place the paravertebral catheter after the incision was made because we like to analyze just the surgical procedure. Doing it during the operation we were focused to understand if placement of the catheter by the surgeon could reduce the time of the procedure itself.

Dr Christian R. Galvez-Padilla (Pittsfield, Mass). Thank you very much for your talk. It was very nice. I am a single thoracic surgeon at a small hospital, so I put in all the paravertebral catheters myself. So it is easy to know that the technique is always the same. I have started to put in the catheters as the second step of the procedure. I perform video-assisted thoracoscopic surgery for all lobectomies; I put in a single 5-mm port for the camera and then put the paravertebral catheter next, to have good pain control from the start of the procedure.

The problem I've seen is that when the catheter is removed, the pain starts, and patients require narcotics. For how long are you leaving in the paravertebral catheter, and what is your experience with pain control when the catheter comes out?

Dr Raveglia. Yes. In this study, we removed it 3 days after surgery. Then we studied the analgesics, local anesthetic, and preferential local anesthetic, and we did not have the impression that we need to leave the catheter in for more time after the 3 days.

But this study was made of 20 patients for the group, as you see. So maybe if there was a bigger group, we might find that we need more time to leave the catheter inside the patient.

Dr Andrew C. Chang (Ann Arbor, Mich). Thanks for doing this study. It is hard to complete a study such as yours. How do you consent patients for this? Do you inform the patient that he or she will receive either an epidural catheter before their operation or a paraspinous catheter placed after the operation is completed?

Because they already know what type of analgesia they're getting, when they report their pain as measured by the visual analog scale, they already may be biased in terms of pain relief. Regarding the methodology, how did you discuss this with the patients?

My second question is regarding the small sample size, which seemed quite small for the $P$ values that you reported. How 
much of an effect were you expecting to find that you would consider statistically significant? That is, when you decided to study 50 patients, how much change in your outcomes, in your visual analog scale were you expecting to see that you would consider significant? Because you had 25 patients in each arm, so to be able to get enough....

Dr Raveglia. We asked for the statistics, specifically how many patients would be necessary to do this kind of study. Our statistician said that this number of patients was sufficient to have statistical data, so that is why we stopped with this number. But we are going on at the hospital, so maybe next year we will have a bigger population.

Dr Chang. And were all patients getting the same operation or were there different types of operations?

Dr Burkhart. Were they all the same operation or different operations?

Dr Raveglia. The thoracotomies were always the same. We decided to treat every patient with the thoracotomy independent of kind of operation, no valve resection or lobectomy for cancer. Thoracotomy was the inclusion criteria. 